Pacific Journal of Mathematics

ON THE IDEAL STRUCTURE OF THE RING OF ENTIRE 


\title{
ON THE IDEAL STRUCTURE OF THE RING OF ENTIRE FUNCTIONS
}

\author{
MELVIN HENRIKSEN
}

1. Introduction. Let $R$ be the ring of entire functions, and let $K$ be the complex field. The ring $R$ consists of all functions from $K$ to $K$ differentiable everywhere (in the usual sense).

The algebraic structure of the ring of entire functions seems to have been investigated extensively first by 0 . Helmer [1].

The ideals of $R$ are herein classified as in [2]: an ideal $I$ is called fixed if every function in it vanishes at at least one common point; otherwise, $I$ is called free. The structure of the fixed ideals was determined in [1]. The structure of the free ideals is determined below.

While examples of free ideals are easily given, transfinite methods seem to be needed to construct maximal free ideals. The latter are characterized below, and it is shown that the residue class field of a maximal free ideal is always isomorphic to $K$; the field theory of E. Steinitz [5] is used.

2. Elementary properties. Many expositions of the elementary properties of entire functions are available; see [3]. Some of these properties will be repeated below for the sake of completeness.

Definition 1. If $f \in R$, let $A(f) \equiv[z \in K \mid f(z)=0]$.

NotE. As in [1], we take $A(f)$ as an "algebraic set." That is, if $z$ is a zero of multiplicity $m$ of $f$, the $z$ appears $m$ times in $A(f)$. The union and intersection of two such sets is taken in the same sense.

DEFinition 2. If $l$ is any subset of $R$, let $A(I) \equiv[A(f) \mid f \in I]$.

(2.1) If $f$ is any nonzero element of $R$, then $A(f)$ is a closed, discrete (and hence finite or countably infinite) subset of $K$ in the natural topology of $K$. Conversely, given any closed, discrete subset $D$ of $K$, there is an $f \in R$ such that $D=A(f)$. Note that any nonempty closed discrete subset of $K$ either is

Received September 18, 1951; this paper is a part of the author's doctoral thesis at the University of Wisconsin under the direction of R. H. Bruck and W. F. Eberlein.

Pacific J. Math. 2 (1952), $179-184$ 
finite or is a sequence $\left\{z_{n}\right\}_{n=1}^{\infty}$ such that $\lim _{n \rightarrow \infty} z_{n}=\infty$.

(2.2) $R$ is an integral domain.

(2.3) If $D=\left\{z_{n}\right\}_{n=1}^{\infty}$ is any closed, discrete subset of $K$, and if $\left\{w_{n}\right\}_{n=1}^{\infty}$ is any sequence of complex numbers, then there is an $f \in R$ such that $f\left(z_{i}\right)=$ $w_{i} ; i=1,2, \ldots$ (see [3] p. 33, Exercise 3).

(2.4) If $A(f)=A(g)$, then $f=g h$, where $h$ is a unit (element with inverse) of $R$.

3. Ideals of $R$. It will be seen below that the nature of the ideals $I$ of $R$ is completely determined by the sets $A(I)$. Since an element of $R$ is a unit if and only if it vanishes nowhere, every function in an ideal vanishes somewhere. Hence the ideals of $R$ are classified as in [2].

DEFinition 3. An ideal $I$ of $R$ is called fixed if $\bigcap_{f} \in{ }_{I} A(f)$ is nonempty. Otherwise $I$ is called a free ideal.

As the definition indicates, the structure of the fixed ideals is very simple. Helmer determined their structure in the course of his investigation of the arithmetic properties of entire functions. He noted that if $S$ is any subset of $R$, then any function $d$ such that

$$
A(d)=\bigcap_{f \in} A(f),
$$

is a greatest common divisor (unique to within a unit factor) of the functions of $S$. Moreover, Helmer showed that if the set $S$ is finite, its elements being $f_{1}, \cdots, f_{n}$, then there exist elements $e_{1}, \cdots, e_{n}$ of $R$ such that $d=e_{1} f_{1}+$ $\cdots+e_{n} f_{n}$. Hence we have:

THEOREM 1 (Helmer). If $f_{1}, \cdots, f_{n}$ is any finite set of elements of $R$, there exists a function $d$ and elements $e_{1}, \ldots, e_{n}$ of $R$ such that

$$
A(d)=\bigcap_{k=1}^{n} A\left(f_{k}\right)
$$

and $d=e_{1} f_{1}+\cdots+e_{n} f_{n}$. Hence any ideal I of $R$ with finite basis is principal, and so is fixed.

Proof. The proof of the first part is given in [1, Theorem 9]. Clearly if the ideal $I$ is generated by $f_{1}, \cdots, f_{n}$, then $\cap_{f} \in_{I} A(f)=A(d)$, where $d$ is a greatest common divisor of $f_{1}, \cdots, f_{n}$. 


\section{COROLLARY 1. No free ideal of $R$ has a finite basis.}

\section{Corolla RY 2. No polynomial can belong to a free ideal.}

Proof. If $I$ is an ideal of $R$ and if $f, g \in I$, then by Theorem $l$ we have $d \in I$ where $A(d)=A(f) \cap A(g)$. Hence any finite number of elements of $I$ have common zeros. But a polynomial $p$ has only a finite number of zeros. Hence if $p \in I$, then all the functions of $I$ would have a common zero, whence $I$ would be a fixed ideal.

Helmer gave an example of a fixed ideal without finite basis (see [1, proof of Theorem 8]).

The example below shows that free ideals exist.

EXAMPLE. Let $\left\{z_{n}\right\}_{n=1}^{\infty}$ be any sequence of complex numbers such that $\lim _{n \rightarrow \infty} z_{n}=\infty$. Let $\left\{S_{N}\right\}=\left\{z_{N}, z_{N+1}, \ldots\right\}$. By (2.1), we can construct for each natural number $N$ an entire function $F_{N}$ vanishing precisely on $S_{N}$. Let $I$ be the ideal generated by the $F_{N}$. It is easily seen that $I$ is a free ideal.

Free ideals are characterized in terms of the families $A(I)$ as in $[2$, Theorem 36]:

THEOREM 2. Let $\left\{A_{a}\right\}_{\alpha} \in_{A}$ (where $A$ is an index set) be a family of sequences of complex numbers $\left\{z_{n}^{(\alpha)}\right\}$ such that $\lim _{n \rightarrow \infty} z_{n}^{(a)}=\infty$, for all $\alpha \in A$. Moreover, suppose that:

(i) The family $\left\{A_{a}\right\}_{a} \in A$ is closed under finite set intersection.

(ii) $\cap_{a} \in A_{a}$ is empty.

If $F_{\alpha}=\left[f \in R \mid f\left(z_{\alpha}\right)=0\right.$, all $\left.z_{\alpha} \in A_{\alpha}\right]$, then the ideal I generated by the families $F_{\alpha}$ is free. Conversely, if $I=\left\{f_{\alpha}\right\}$ is any free ideal of $R$, and $A_{\alpha}=\left[z \in K \mid f_{\alpha}(z)=\right.$ $0]$, then the family $\left\{A_{\alpha}\right\}$ satisfies (i) and (ii) above.

Proof. Suppose $I$ is defined as above. Since (i) holds, $I$ is closed under subtraction. If $f \in I, g \in R$, then $f$ is in some $F_{\alpha}$, whence $f g$ is in the same $F_{a}$. So $I$ is an ideal, and (ii) ensures that it is free.

Conversely, if $I$ is any free ideal, Theorem $l$ ensures that (i) holds, and (ii) follows from the definition of a free ideal.

4. Maximal ideals and their residue class fields. If $z_{0}$ is any fixed element of $K$, let $l\left(z_{0}\right) \equiv\left[f \in R \mid f\left(z_{0}\right)=0\right]$. Clearly $l\left(z_{0}\right)$ is a fixed ideal of $R$. Moreover, the mapping $f(z) \rightarrow f\left(z_{0}\right)$ is clearly a homomorphism of $R$ upon $K$ whose kernel is $I\left(z_{0}\right)$. Hence $I\left(z_{0}\right)$ is a maximal fixed ideal. Conversely, if $I$ is a fixed ideal and if $\cap_{f \in I} A(f)$ contains two points $z_{1}, z_{2}$ (not necessarily distinct), then $I$ is properly contained in $I\left(z_{1}\right)$ or $I\left(z_{2}\right)$. Hence we have proved: 
THEOREM 3. Every maximal fixed ideal of $R$ is of the form

$$
K\left(z_{0}\right)=\left[f \in R \mid f\left(z_{0}\right)=0\right]
$$

for some $z_{0} \in K$. Moreover, the residue class field of every maximal fixed ideal is the complex field $K$.

The maximal free ideals are not so simple in structure. They may, however, be characterized as follows:

THEOREM 4. A free ideal $M$ of $R$ is maximal if and only if $A(M)$ satisfies:

(iii) If $D=\left\{z_{n}\right\}_{n=1}^{\infty}$ is any infinite, closed, discrete set of $K$ such that $D \cap A(f)$ is nonempty for every $f \in M$, then $D \in A(M)$.

Proof. Suppose $M$ is a free ideal and (iii) holds. If $M$ is not maximal, then there is an ideal $N$ properly containing $M$. Suppose $g \in N$ and apply (i) of Theorem 2 to $A(N)$. Then $A(g) \cap A(f)$ is nonempty for every $f \in N$, and hence for every $f \in M$. Hence $g \in M$ by (iii). Hence $M$ is a maximal free ideal.

Conversely, suppose $M$ is a maximal free ideal. If there were an infinite, closed, discrete set $D$ violating (iii), then any $g \in R$ such that $A(g)=D$ would together with $M$ generate an ideal $N$ properly containing $M$. Hence (iii) must hold.

Note. This result is similar to a theorem of Hewitt on maximal ideals of rings of real valued continuous functions; see [2, Theorem 36].

Since maximal free ideals are complicated in structure, it is natural to expect the same of their residue class fields. First we show:

THEOREM 5. If $M$ is a maximal free ideal, then $R / M$ contains a subfield isomorphic to the field $R(z)$ of all rational functions of a complex variable $z$.

Proof. By Corollary 2 of The orem $1, M$ can contain no polynomial. Hence if $p_{1}, p_{2}$ are two distinct polynomials, then $p_{1} \not \equiv p_{2}(\bmod M)$. Hence $R / M$ contains as a subring all polynomials in $z$. So $R / M$ contains $R(z)$ as a subfield.

COROLLARY. The field $K$ is subfield of $R / M$. If $R$ is considered as an algebra over $K$, then the residue class field $R / M$ may be considered as a division algebra containing $K$ as a proper subfield.

Proof. If $R$ is considered to be an algebra over $K$, the homomorphism of $R$ upon the quotient algebra $R / M$ is assumed to keep complex numbers fixed. Hence $K$ is a proper division subalgebra of $R / M$.

If one does not insist that the complex numbers stay fixed under a homo- 
morphism of $R$ upon $R / M$, we have:

THEOREM 6. If $M$ is a maximal free ideal, then $R / M$ is isomorphic (as a ring) to $K$.

We shall establish two lemmas before proving the theorem.

LEMMA 1. The field $R / M$ is algebraically closed.

Proof. Note first that if $f \in M$, then $M$ contains all functions vanishing on the distinct points of $A(f)$. This is true since the maximality of $M$ ensures that $M$ contains a function $g$ with simple zeros at the distinct points of $A(f)$; and by (2.4) it contains all such functions. Now let

$$
\Phi(z, X)=f_{0}(z)+f_{1}(z) X+\cdots+f_{n}(z) X^{n}
$$

be any polynomial with coefficients $f_{0}, f_{1}, \cdots, f_{n} \in R$, where $f_{n}$ is not in $M$, $n>0$. Choose any sequence $\left\{z_{k}\right\} \in A(M)$. Now for any fixed $k$, the function $\Phi\left(z_{k}, X\right)$ is a polynomial with coefficients in $K$ and hence has $n$ complex roots. Choose any such root and call it $r_{k}$. Then construct, by (2.3), a function $g \in R$ such that $g\left(z_{k}\right)=r_{k}(k=1,2,3, \cdots)$. Clearly, by the above, $\Phi(z, g(z)) \equiv 0$ $(\bmod M)$. Hence $R / M$ is algebraically closed.

LEMMA 2. The field $R / M$ contains $c$ elements, where $c$ is the cardinal number of the continuum.

Proof. Since $K$ contains a countable dense subset, there are only $c$ continuous functions, and hence only $c$ entire functions. Hence $R / M$ has at most $c$ elements. But all complex numbers are incongruent $(\bmod M)$, so $R / M$ has at least $c$ elements. Hence $R / M$ has precisely $c$ elements.

Proof of Theorem 6. Steinitz has shown [5, p. 125, Section 6] that any algebraically closed field of characteristic 0 and of degree of transcendency $c$ over its prime field is isomorphic to $K$. Since $R / M$ contains $K$, it has degree of transcendency at least $c$. By Lemma $2, R / M$ can have degree of transcendency at most $c$. Hence $R / M$ has degree of transcendency $c$. By Lemma $1, R / M$ is algebraically closed. Hence $R / M$ is isomorphic to $K$.

5. Topological considerations. In [4], Schilling investigated the ring $R$ of entire functions as a topological ring under the topology of uniform convergence on compact sets. He showed [4, p.949, Lemma 3] that any closed ideal of $R$ is principal. Hence, in particular, no free ideal is closed. He also stated [4, p. 952, Lemma 6] that a maximal ideal $M$ of $R$ is nonclosed if and only if $R / M$ is a proper extension of $K$. This is in apparent contradiction with our Theorem 6 . 
This apparent discrepancy is easily resolved. Although it is nowhere explicitly stated, Schilling considered $R, R / M$, and $K$ as algebras over $K$. He then proved the equivalent of our Theorem 5 . Thus $R / M$ is a commutative division algebra containing $K$ properly, which is isomorphic as a ring to $K$.

\section{REFERENCES}

1. O. Helmer, Divisibility properties of integral functions, Duke Math. J. 6(1940), $345-356$.

2. E. Hewitt, Rings of real valued continuous functions. I, Trans. Amer. Math. Soc. 64 (1948), 45-99.

3. K. Knopp, Theory of Functions, vol. 2, Dover Publications, New York, 1947.

4. O. F. G. Schilling, Ideal theory on open Riemann surfaces, Bull. Amer. Math. Soc. 52 (1946), 945 - 963.

5. E. Steinitz, Algebraische Theorie der Körper, Walter de Gruyter and Co., Berlin, 1930.

UNIVERSITY OF WISCONSIN AND

University of Alabama 


\section{PACIFIC JOURNAL OF MATHEMATICS}

\section{EDITORS}

R. M. RoBINSON

University of California Berkeley 4, California

\section{*R. P. Dilworth}

Califomia Institute of Technology Pasadena 4, California

\section{E. F. BECKENBACH, Managing Editor}

University of California

Los Angeles 24, California

*During the absence of Herbert Busemann in 1952.

\section{ASSOCIATE EDITORS}

\author{
R. P. DILWORTH \\ HERBERT FEDERER \\ MARSHALL HALL
}

\author{
P. R. HALMOS \\ HEINZ HOPF
}

R. D. JAMES

\author{
BØRGE JESSEN \\ PAUL LÉVY \\ GEORGE PÓLYA
}

\author{
J. J. STOKER \\ E. G. STRAUS
}

KÖSAKU YOSIDA

\section{SPONSORS}

UNIVERSITY OF BRITISH COLUMBIA

CALIFORNIA INSTITUTE OF TECHNOLOGY

UNIVERSITY OF CALIFORNIA, BERKELEY

UNIVERSITY OF CALIFORNIA, DAVIS

UNIVERSITY OF CALIFORNIA, LOS ANGELES

UNIVERSITY OF CALIFORNIA, SANTA BARBARA

OREGON STATE COLLEGE

UNIVERSITY OF OREGON

\author{
UNIVERSITY OF SOUTHERN CALIFORNIA \\ STANFORD UNIVERSITY \\ WASHINGTON STATE COLLEGE \\ UNIVERSITY OF WASHINGTON \\ AMERICAN MATHEMATICAL SOCIETY \\ NATIONAL BUREAU OF STANDARDS, \\ INSTITUTE FOR NUMERICAL ANALYSIS
}

Mathematical papers intended for publication in the Pacific Journal of Miathematics should be typewritten (double spaced), and the author should keep a complete copy. Manuscripts may be sent to any of the editors. All other communications to the editors should be addressed to the managing editor, E. F. Beckenbach, at the address given above.

Authors are entitled to receive 100 free reprints of their published papers and may obtain additional copies at cost.

The Pacific Journal of Mathematics is published quarterly, in March, June, September, and December, by the University of California, Berkeley 4, California. The price per volume (4 numbers) is $\$ 8.00$; single issues, $\$ 2.50$. Special price to individual faculty members of supporting institutions and to individual members of the American Mathematical Society: $\$ 4.00$ per volume; single issues, $\$ 1.25$.

Subscriptions, orders for back numbers, and changes of address should be sent to the publishers, University of California Press, Berkeley 4, California.

Printed at Ann Arbor, Michigan. Entered as second class matter at the Post Office, Berkeley, California.

\section{UNIVERSITY OF CALIFORNIA PRESS • BERKELEY AND LOS ANGELES}




\section{Pacific Journal of Mathematics}

\section{Vol. 2, No. $2 \quad$ February, 1952}

L. Carlitz, Some theorems on Bernoulli numbers of higher order...

Watson Bryan Fulks, On the boundary values of solutions of the heat equation........................................ 141

John W. Green, On the level surfaces of potentials of masses with fixed center of gravity................................... 147

Isidore Heller, Contributions to the theory of divergent series .......... 153

Melvin Henriksen, On the ideal structure of the ring of entire functions . . . 179

James Richard Jackson, Some theorems concerning absolute neighborhood retracts........................................ 185

Everett H. Larguier, Homology bases with applications to local connectedness ................................... 191

Janet McDonald, Davis's canonical pencils of lines ................ 209

J. D. Niblett, Some hypergeometric identities . . . . . . . . . . . . . . . . . . 219

Elmer Edwin Osborne, On matrices having the same characteristic equation...................................... 227

Robert Steinberg and Raymond Moos Redheffer, Analytic proof of the

Lindemann theorem ................................. 231

Edward Silverman, Set functions associated with Lebesgue area ......... 243

James G. Wendel, Left centralizers and isomorphisms of group algebras . . 251

Kosaku Yosida, On Brownian motion in a homogeneous Riemannian space. 